\title{
Polymer-Stretching-Regulated Microscopic Photoluminescence of Vibration-Induced Emission (VIE) Molecules
}

Fan Gu, Yuanhao Li, Tao Jiang, Jianhua Su, Xiang Ma* and He Tian

Key Laboratory for Advanced Materials and Feringa Nobel Prize Scientist Joint Research Center, Frontiers Science Center for Materiobiology and Dynamic Chemistry, School of Chemistry and Molecular Engineering, East China University of Science and Technology, Meilong Road 130, Shanghai 200237, China.

*Correspondence to: maxiang@ecust.edu.cn

Abstract: Photoluminescence materials play an inseparable role in the application of polymer systems. However, intrinsic polymer systems have rarely been intuitively interpreted based on photoluminescence regulation. A novel photoluminescence mechanism called vibrationinduced emission (VIE) has recently drawn great attention due to its multicolor fluorescence from a single molecular entity. Based on the unique fluorescent properties of VIE molecules, we doped 9,14-diphenyl-9,14-dihydrodibenzo[a,c]-phenazine (DPAC) and its derivative DPAC-CN in two stretchable polymers, poly( $\varepsilon$-caprolactone) and ethylene vinyl acetate (EVA) copolymer, to explore the important relationship between luminophores and polymer systems. This research focused on the multicolor photoluminescence of the obtained blend films that resulted from stretching exertions and temperature responses. The successive conformational alterations of VIE molecules endowed continuous photoluminescent changes. Meanwhile, the multicolor variations also provided specific visual evidence regarding the amplified tensile stresses and microstructural changes in the polymer. This demonstration will therefore provide advantageous insights into the development of functional optical materials.

\section{Introduction}

Recently, photoluminescence mechanisms with luminescence-tunable behaviors and stable emission properties have ushered in rapid development stages in both academic exploration ( 1 , 2) and fundamental inventions $(3,4)$. Based on this, the development of many potential applications, such as detection (5), data storage (6), anti-counterfeiting $(7,8)$, and displays $(9$ - 
12), has created great value based on the dramatic illumination of functional optical materials. However, the optical function achieved by photoluminescence luminophores is not always established by the luminophores themselves $(13,14)$. Owing to the unique properties of materials, most researchers have realized innovative photoluminescence materials that rely on the combination of luminophores and polymers by doping and polymerization (15-19). Thus, the application of specific polymers has become prominent in the area of photoluminescence materials (20-22). Luminophores has undoubtedly created more possibilities regarding the assistance of polymer systems. Nonetheless, the photoluminescence mechanism has rarely been utilized in the detection and analysis of polymer systems. There are still challenges in intrinsic amplification of polymers by the advantages of photoluminescence regulation. Due to the limitations of luminophores, many luminescence systems exhibit single-color emissions or multicolor emissions under certain conditions $(23,24)$. This induces restrictions on the further development of functional optical materials in polymer systems. Therefore, it is of great importance to fully utilize the representative behavior of photoluminescence regulation to realize internal visualization in polymer systems.

Vibration-induced emission (VIE) (25-27), as an emerging photoluminescence mechanism, has drawn great attention in recent years owing to its tunable multicolor mission between different successive configurations. This mechanism has been attributed to the saddle-like VIE molecules of 9,14-diphenyl-9,14-dihydrodibenzo[a,c]-phenazine and its derivatives with consecutive conformations from bent to planar (28-31), resulting in appealing multicolor mission (blue and orange red) from a single molecular entity. Under different external environmental conditions, such as solvent polarity $(32,33)$, viscosity $(34,35)$ and temperature (36), VIE molecules display excellent photoluminescence properties with favorable reversibility and controllable regulations $(37,38)$. Based on this phenomenon, we proposed a distinct strategy involving the exertion of an external stretching force to explore and report the very special and interesting photoluminescent characteristics of VIE molecules in poly $(\varepsilon$ caprolactone) and ethylene vinyl acetate (EVA) polymer systems. As biodegradable polymers, poly( $\varepsilon$-caprolactone) and EVA materials that have good extensibility properties have been generally accepted to form homogeneous mixtures with other compounds in the melt and 
solution. The physical parameters of poly( $\varepsilon$-caprolactone) and EVA have been extensively explored in many previous studies (39-42). However, the measurements of intrinsic alterations in polymer systems still require the use of sophisticated instrument detection, which has not been represented by an intuitional method so far. Therefore, we attempted to visualize and amplify the microcosmos of poly(e-caprolactone) and EVA by utilizing the multicolor luminescent properties of VIE molecules. We believe that the integration of VIE theory will contribute to gaining a profound understanding of polymer systems, as well as discovering future applications that further enrich functional optical materials.

In the present work, we synthesized two VIE molecules, 9,14-diphenyl-9,14dihydrodibenzo[a,c]-phenazine $\quad$ (DPAC) and 9,14-diphenyl-9,14dihydrodibenzo[a,c]phenazine-11-carbonitrile (DPAC-CN). These two VIE molecules were simply doped into two polymer systems respectively to explore the intrigue photoluminescence mechanisms. Polymer systems including poly( $\varepsilon$-caprolactone) and EVA were chosen because of their excellent stretchability and flexibility; such properties are the reason that the VIE molecules exhibited different conformations from bent to planar and represented dramatic photoluminescent behavior in these polymers. The novel blend films we obtained not only exhibited diverse fluorescence emissions but also precise recording of exerted tensile stress and visualized changes in the microstructure and crystallinity of polymer systems according to the gradual conformation changes. Additionally, the temperature responses in both polymer systems were also extensively explored. The obtained material with VIE molecules was even utilized as a temperature detector for the recognition of body temperature. Therefore, the two systems with VIE molecules made significant contributions to the amplification of microcosmos in polymers through consecutive photoluminescence emissions.

\section{Microcosmic disclosure in a doping system of poly(e-caprolactone) by VIE photoluminescence regulation}

The VIE molecules of DPAC and DPAC-CN possessed multicolor emissions owing to the consecutive conformations that varied with different surroundings (detailed synthetic processes are reported in the Supplementary Materials and figs. S1 to S6). As shown in fig. S7, DPAC and DPAC-CN emit blue fluorescence in the solid state with respective fluorescence quantum 
yields (QYs) of 5.9\% and 3.8\%. The respective red fluorescence emission in toluene solution is exhibited in fig. S8. Along with reacting to variations in the external surroundings, such as the solvent polarity, viscosity and temperature, the VIE molecules showed diverse emissions from blue to orange red through a gradual process; such emissions are inseparable from the successive changes in their conformations. The related mechanism has been fully studied in our previous reports (28-36). For further exploration, this research focused on microcosmic disclosure in polymer systems by photoluminescence regulation of VIE molecules (Fig. 1).

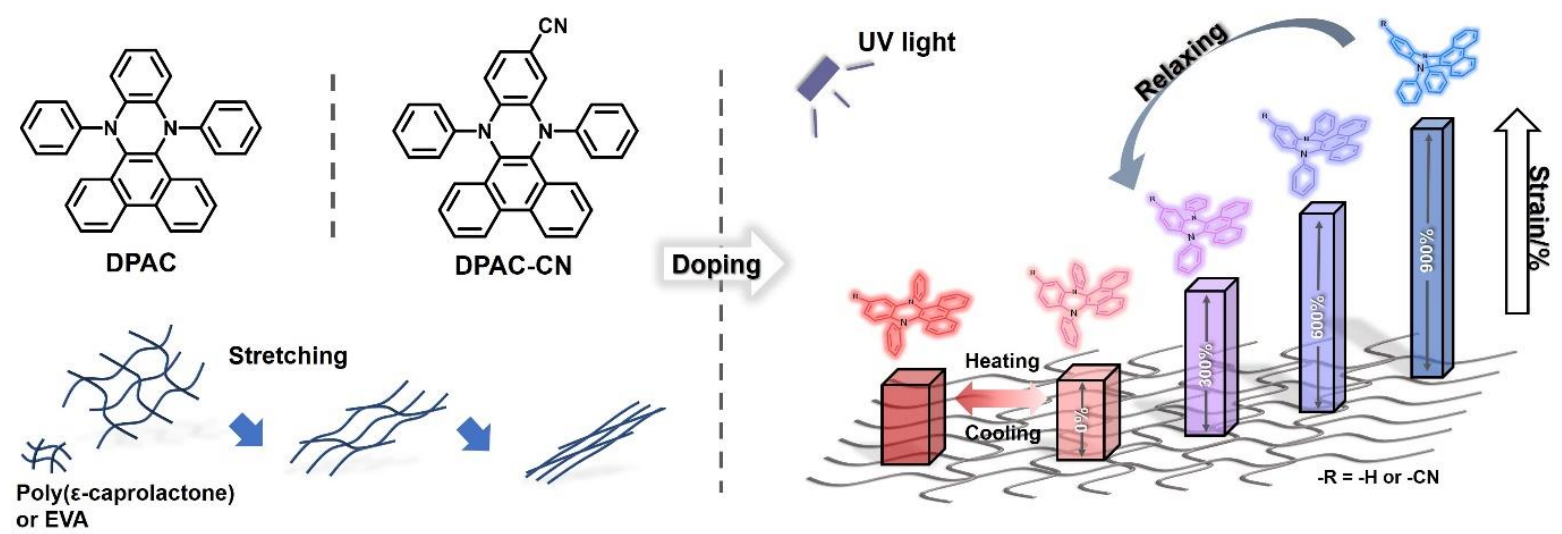

Fig. 1. Illustration of the film preparation and the photoluminescence mechanisms in different stretching states. Preparation of the blend films in two polymer systems and the chemical structures of DPAC and DPAC-CN in different conformations, from bent to planar, upon stretching and heating in the excited states.

P(DPAC) and P(DPAC-CN) films were obtained by doping DPAC and DPAC-CN in poly $(\varepsilon-$ caprolactone) (Fig. 1). The detailed preparation processes are provided in the Supplementary Materials. To eliminate the influence of poly( $\varepsilon$-caprolactone) on the luminescence behaviors, fluorescence spectra and absorption spectra of the poly( $\varepsilon$-caprolactone) film were obtained under the same test conditions as $\mathrm{P}(\mathrm{DPAC})$ and $\mathrm{P}(\mathrm{DPAC}-\mathrm{CN})$, as shown in figs. S9 and S10. It was demonstrated that the poly( $\varepsilon$-caprolactone) film had no effect on the photoluminescence in either the original state or the stretched state. Based on this result, we prepared films at different doping ratios with increasing VIE molecule concentrations (wt\%), namely, P(DPAC0.1\%), P(DPAC-0.3\%), P(DPAC-0.5\%), P(DPAC-1.5\%), P(DPAC-2.5\%), P(DPAC-5\%), 
P(DPAC-CN-0.1\%), P(DPAC-CN-0.3\%), P(DPAC-CN-0.5\%), P(DPAC-CN-1.5\%), $\mathrm{P}(\mathrm{DPAC}-\mathrm{CN}-2.5 \%)$, and P(DPAC-CN-5\%).

The fabricated films exhibited different photoluminescent behaviors with increasing VIE molecule concentrations in poly( $\varepsilon$-caprolactone). Taking $\mathrm{P}(\mathrm{DPAC})$ as an example, the resulting normalized fluorescence spectra corresponded to multicolor fluorescence from light pink to yellow green, as shown in Fig. 2 (A and C). The short wavelength emissions at different ratios became increasingly redshifted from 425 to $470 \mathrm{~nm}$. However, the long wavelength emissions were all located at $600 \mathrm{~nm}$ and did not change significantly; this indicated that the addition of DPAC lead to conjugation resulting from intermolecular stacking but did not increase the conjugation of the planar configuration in the excited state. Furthermore, the long wavelength intensity between $\mathrm{P}(\mathrm{DPAC}-0.1 \%)$ and $\mathrm{P}(\mathrm{DPAC}-0.5 \%)$ rose slightly according to the normalized fluorescence spectra, meaning that the DPAC molecules were dispersive with few restrictions at low concentrations. Conversely, the long wavelength intensity declined with further increases in DPAC\% from $\mathrm{P}(\mathrm{DPAC}-0.5 \%)$ to $\mathrm{P}(\mathrm{DPAC}-5 \%)$, owing to the limitations caused by intermolecular stacking in the excited state. To demonstrate this behavior, the absorption spectra of $\mathrm{P}(\mathrm{DPAC})$ and $\mathrm{P}(\mathrm{DPAC}-\mathrm{CN})$ films are shown in fig. S11. The redshifts of the absorption peaks provided strong evidence for increases in intermolecular stacking and conjugation. Similar multicolor variations also existed in P(DPAC-CN), as shown in Fig. 2 (B and $\mathrm{C})$. With the electron withdrawing effect of $-\mathrm{C} \equiv \mathrm{N}, \mathrm{P}(\mathrm{DPAC}-\mathrm{CN})$ exhibited more redshifted short wavelength emissions from 465 to $495 \mathrm{~nm}$ than P(DPAC). Owing to the effect of intermolecular stacking, the fluorescence QYs of the P(DPAC) and P(DPAC-CN) films, as measured and shown in Fig. 2D, did not change significantly with additional doping concentrations but exhibited stronger photoluminescence properties than the original VIE molecules. 

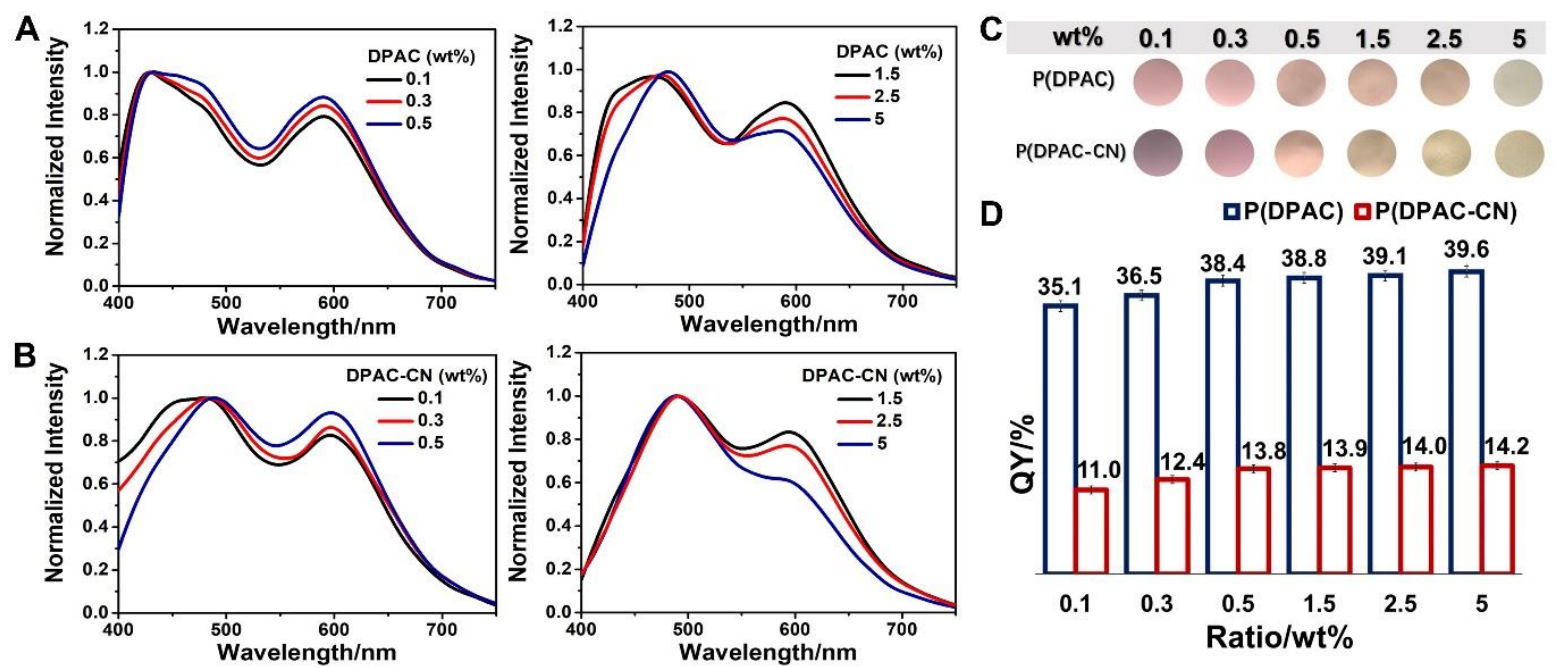

Fig. 2. Photoluminescent properties at different doping ratios. Normalized fluorescence spectra of (A) $\mathrm{P}(\mathrm{DPAC})$ and (B) $\mathrm{P}(\mathrm{DPAC}-\mathrm{CN})$ films with different doping ratios. (C) Representative photographs of $\mathrm{P}(\mathrm{DPAC})$ and $\mathrm{P}(\mathrm{DPAC}-\mathrm{CN})$ films with different doping ratios under $365 \mathrm{~nm}$ excitation. (D) Fluorescence QYs of P(DPAC) and P(DPAC-CN) films with different doping ratios $\left(\lambda_{\mathrm{ex}}=365 \mathrm{~nm}\right)$.

Inspired by the various photoluminescent properties in $\mathrm{P}(\mathrm{DPAC})$ and $\mathrm{P}(\mathrm{DPAC}-\mathrm{CN})$ films, we further studied the tension-visualization behaviors in these blend films with certain external tensile stresses. Despite low elasticity, due to the commendable extensibility of poly $(\varepsilon-$ caprolactone), the blend films with VIE molecules were stretched at a constant speed $(10 \mathrm{~mm}$ $\min ^{-1}$ ) while the precise corresponding tensile stresses were recorded. As shown in Fig. 3, stretching experiments were conducted on P(DPAC-0.1\%), P(DPAC-0.5\%), P(DPAC-5\%), $\mathrm{P}(\mathrm{DPAC}-\mathrm{CN}-0.1 \%), \mathrm{P}(\mathrm{DPAC}-\mathrm{CN}-0.5 \%)$, and $\mathrm{P}(\mathrm{DPAC}-\mathrm{CN}-5 \%)$ to record the consecutive photoluminescence changes in this system. The stress-strain curves of the films in fig. S12 were markedly parallel and agreed with the poly( $\varepsilon$-caprolactone) curve in both elongation and breaking strength. The corresponding average mechanical strength was $28 \mathrm{MPa}$ and the satisfactory stretchability was $900 \%$, implying that a small amount of VIE molecules did not induce changes in the nature of poly( $\varepsilon$-caprolactone). Moreover, the X-ray diffraction (XRD) spectra and differential scanning calorimetry (DSC) thermograms also demonstrated this point owing to the small changes among poly( $\varepsilon$-caprolactone), P(DPAC-0.5\%) and P(DPAC-0.5\%) in the stretched state, as exhibited in figs. S13 and S14. 
Specifically, we were surprised to discover that blend films doped with VIE molecules emitted distinct fluorescence during the stretching process upon $365 \mathrm{~nm}$ excitation, as compared to the original and stretched films without $365 \mathrm{~nm}$ excitation in fig. S15. This variation in fluorescence color would significantly match with the continuous stretching process. As shown in Fig. 3 (A to C), the blend films of P(DPAC-0.1\%), P(DPAC-0.5\%) and $\mathrm{P}(\mathrm{DPAC}-5 \%)$, as prepared with rectangular shapes $(20 \mathrm{~mm} \times 10 \mathrm{~mm})$, were stretched separately, in parallel, at a uniform speed. Then, the normalized fluorescence spectra of the stretched parts were recorded for elongations from 0 to $900 \%$. The original states of these films showed two characteristic emission peaks $(425 \mathrm{~nm}$ and $600 \mathrm{~nm}$ ) that were properly assigned to the DPAC doped in poly( $\varepsilon$-caprolactone). The amplitude of the broad emission peak located at $425 \mathrm{~nm}$ gradually decreased and the peak was finally replaced by the $480 \mathrm{~nm}$ emission peak, as shown in Fig. 3C. This phenomenon related to stretching progress was similar to the phenomenon related to increasing VIE molecule concentration, convincingly demonstrating that stretching of the films induced narrow intermolecular distances of VIE molecules. Furthermore, the intensity of the long wavelength emission decreased gradually with slow elongation, meaningfully reinforcing the successive configuration limitations of VIE molecules in the excited state brought by stretching. The restriction that weakened the red fluorescence emission of blend films finally eliminated all but the blue emission, implying stronger restrictions of molecular configuration induced by stretching. This was demonstrated by the stretching images of $\mathrm{P}(\mathrm{DPAC}-0.5 \%)$ shown in Fig. 3G. The progressive increase in the fluorescence QYs with deformations at 100\%, 400\% and 900\%, as shown in fig. S16, might also account for the facilitation of narrow intermolecular distances. Similarly, P(DPAC-CN) exhibited fluorescence color conversions from light orange to green after stretching (Fig. 3, D to F, fig. S15 and movie S1). Increases also occurred in the QYs of P(DPAC-CN-0.5\%). This dramatic fluorescent behavior in blend films was also captured again once the stretched films were redissolved in dichloromethane (DCM) and dried off, demonstrating the excellent reversibility enabled by $\mathrm{P}(\mathrm{DPAC}-0.5 \%)$ (fig. S17). 

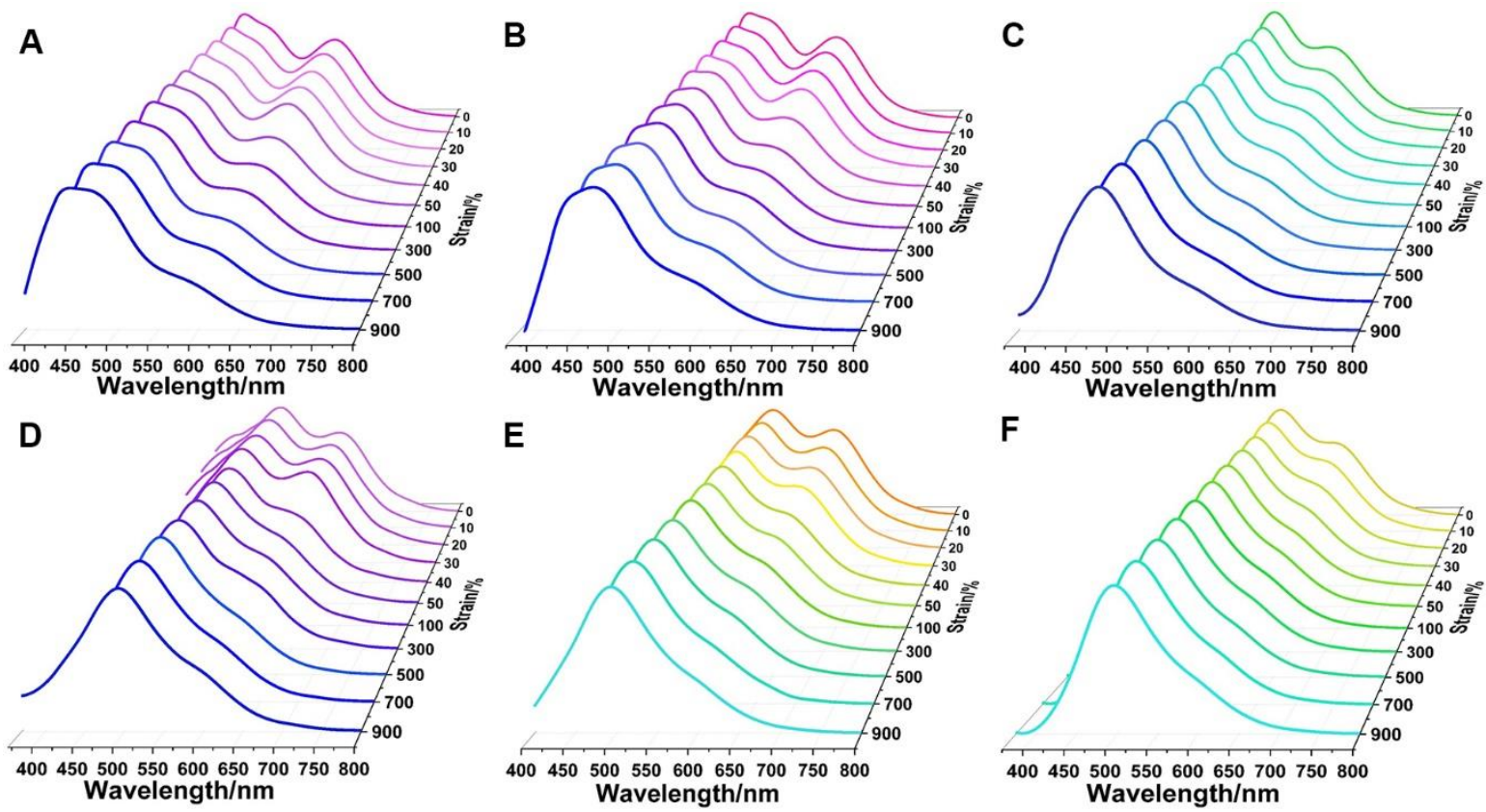

400450500550600650700750800 Wavelength/nm
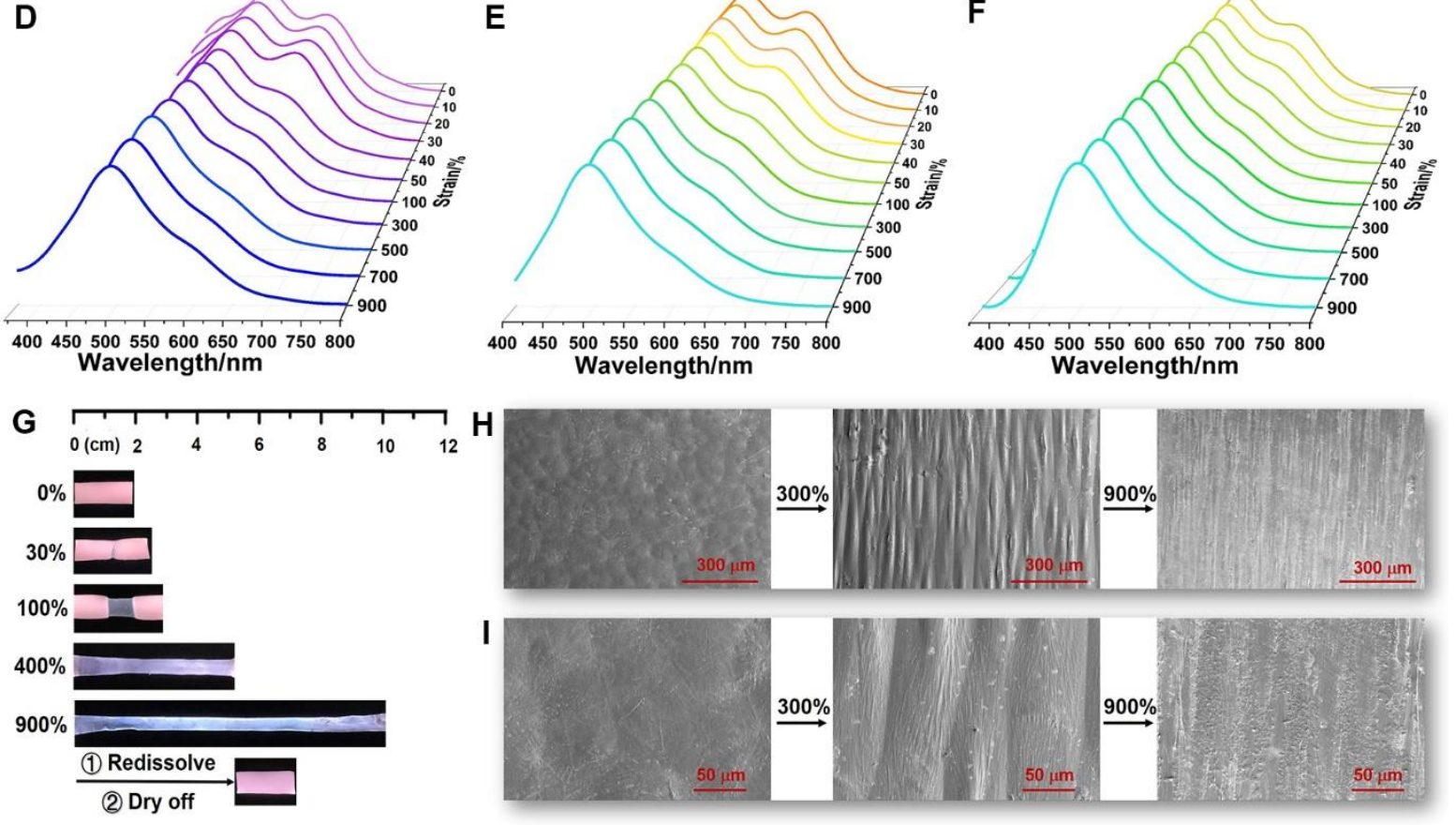

Fig. 3. Stretching properties at different elongations. Normalized fluorescence spectra of (A) P(DPAC-0.1\%), (B) P(DPAC-0.5\%), (C) P(DPAC-5\%), (D) P(DPAC-CN-0.1\%), (E) $\mathrm{P}(\mathrm{DPAC}-\mathrm{CN}-0.5 \%)$, and (F) $\mathrm{P}(\mathrm{DPAC}-\mathrm{CN}-5 \%)\left(\lambda_{\mathrm{ex}}=365 \mathrm{~nm}\right)$. The line color exhibited is connected to the luminescence color of the blend films. (G) Photoluminescence images of P(DPAC-0.5\%) at different stretching states under $365 \mathrm{~nm}$ excitation. (H to I) SEM images of the P(DPAC- $0.5 \%)$ film before and after stretching, with scale bars of $300 \mu \mathrm{m}$ and $50 \mu \mathrm{m}$.

To evaluate the restricted effect of poly( $\varepsilon$-caprolactone), we performed absorption measurements of $\mathrm{P}(\mathrm{DPAC}-0.5 \%)$ and $\mathrm{P}(\mathrm{DPAC}-\mathrm{CN}-0.5 \%)$ upon stretching, as shown in fig. S18. The existence of blueshifted absorption peaks in the original and stretched states implied a decrease in the luminophore concentration, indicating further narrow distances of VIE molecules in polymer networks. The limitations induced by stretching mainly occurred in the excited state of VIE molecules and completely decreased the long wavelength intensity. This 
decrease confirmed that the changes in the surroundings of the obtained films, as observed between the original states and stretched states, played a crucial role in the fluorescence emission of VIE molecules. The scanning electron microscopy (SEM) images of P(DPAC$0.5 \%$ ) (Fig. 3, H and I) provided strong evidence to support the idea that more restrictions were induced by stretching. With further elongations at $300 \%$ and $900 \%$, the surfaces of the films showed obvious changes in the microstructure and crystallinity of poly( $\varepsilon$-caprolactone). This phenomenon has been identified in previous research $(39,40)$. However, owing to the different conformations realized by the VIE molecules, the changes in microstructure and crystallinity were visualized based on their multicolor photoluminescent behaviors under ultraviolet (UV) light irritation. This enables a profound understanding of how to amplify microscopic changes in polymers through photochemistry.
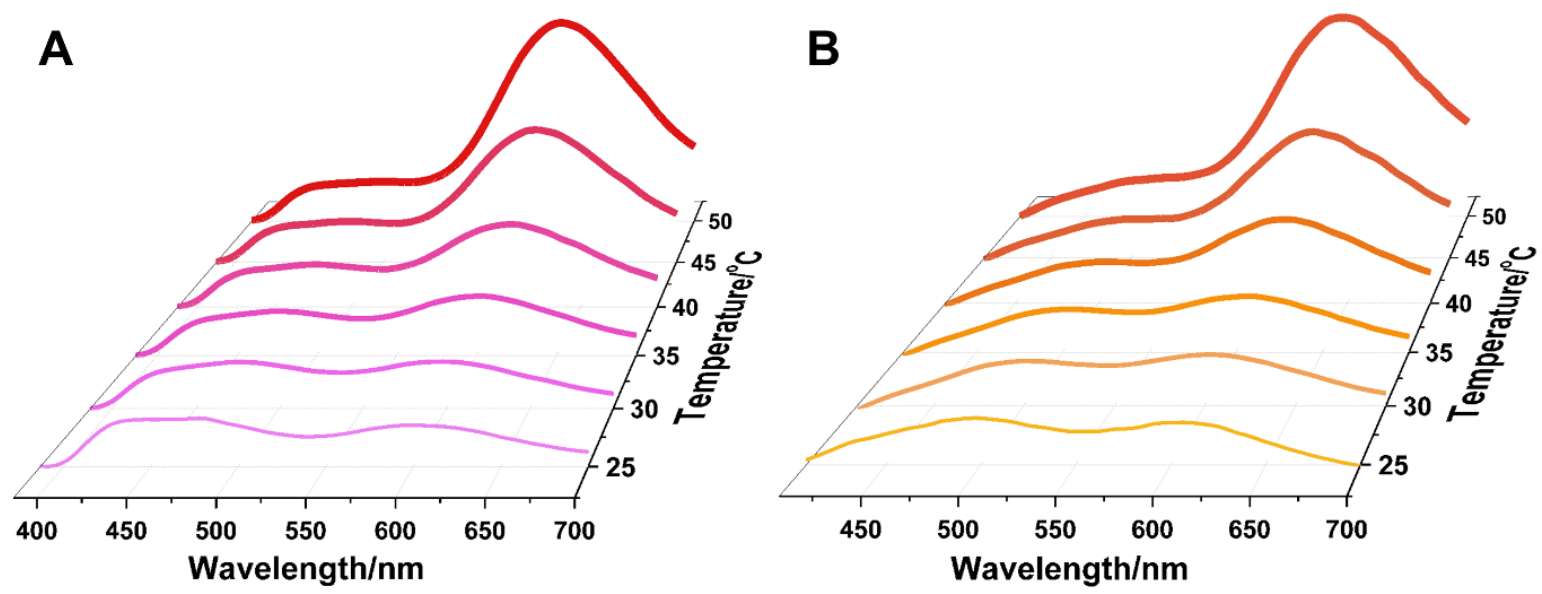

Fig. 4. Temperature responses in the doping system of poly(\&-caprolactone). Normalized fluorescence spectra of (A) P(DPAC-0.5\%) and (B) P(DPAC-CN-0.5\%) with increasing temperatures from 25 to $50^{\circ} \mathrm{C}$.

Additionally, the films obtained with dramatic fluorescent behaviors not only demonstrated the microstructure and crystallinity changes in poly(e-caprolactone) but also recorded the continuous force value through their diverse fluorescence emissions, realizing tension visualizations. Owing to the differences in the photoluminescent properties between DPAC and DPAC-CN, the resulting fluorescence color conversions differed in Commission Internationale de L'Eclairage (CIE) chromaticity coordinate value, as shown in figs. S19 and S20. In addition, every force value was matched with every CIE coordinate value for the exact 
elongation ratios, as shown in tables S1 and S2. The VIE theory enabled consecutive configuration changes, which supported successive multicolor fluorescence upon increasing elongation. This could be a significant symbol for use in the realization of intercalibration between photoluminescence and mechanical properties.

Furthermore, the blend films we obtained demonstrated sensitive temperature responses from 25 to $50^{\circ} \mathrm{C}$. The normalized fluorescence spectra of P(DPAC-0.5\%) and P(DPAC-CN$0.5 \%$ ) at different temperatures were used as examples, as shown in Fig. 4 (A and B). With increasing temperatures, the long wavelength intensities increased dramatically, and the fluorescence colors of films changed from light pink (light orange for P(DPAC-CN-0.5\%)) to orange red. The multicolor changes were recorded in movie S2. Such changes occurred because the higher temperature activated the intramolecular motions of VIE molecules and generated more planar conformations in the excited state (36). The temperature responses of the blend films were also matched to every CIE coordinate value with increasing temperature, as shown in fig. S21 and table S3. Owing to the increases in intramolecular motions, the QYs of the fluorescence emissions decreased gradually. Upon heating and cooling for 8 cycles, this process exhibited favorable reversibility without substantial changes in the fluorescent behavior, as shown in fig. S22. Compared with the limitations induced by the stretching process, the increasing temperature provided more flexible space for the VIE molecules; in contrast to stretching, the higher number of red fluorescence emissions also demonstrated the more flexible surroundings in polymer system upon temperature increase.

\section{Microcosmic disclosure in a doping system of EVA by VIE photoluminescence regulation}

In addition to the controllable tension visualization of VIE molecules in the poly $(\varepsilon-$ caprolactone) system, similar properties were also achieved in the EVA system. As an advanced copolymer, EVA has been widely applied in industrial applications such as flexible packaging, membranes, photovoltaic cells, and adhesives $(41,42)$. Owing to its similar tensile properties but satisfactory elasticity to poly( $\varepsilon$-caprolactone), EVA was also chosen as a polymer matrix for VIE molecules. The EVA materials were colorless transparent films, as shown in fig. S23. To eliminate natural interferences from EVA, stress-strain tests were conducted and XRD spectra were obtained for EVA-(DPAC), EVA-(DPAC-CN) and EVA; 
such characterizations indicated little alteration of the materials with VIE molecules, as shown in figs. S24 and S25. The absorption spectra and fluorescence spectra of EVA itself also confirmed this conclusion (figs. S10 and S26). Nonetheless, EVA possessed not only a satisfactory stretchability of $600 \%$ but also a favorable elasticity (fig. S27), enabling the possibility of recovering the photoluminescent changes. As shown in Fig. 5A and fig. S28, EVA-(DPAC) and EVA-(DPAC-CN) presented similar multicolor fluorescence behaviors from light pink to pale blue (light orange to light green for EVA-(DPAC-CN)) with elongation changes from $0-600 \%$. The amplitude of the long wavelength peak gradually decreased owing to the limitations of molecular conformation that resulted from stretching. Moreover, the long wavelength peak increased again once the tensile stress was gradually relaxed, demonstrating the release of the restriction of VIE molecules in the excited state. The fluorescence color also returned to the original state, as shown in movie S3. The absorption spectra in fig. S29 also verifies the recovery of DPAC and DPAC-CN. SEM images of EVA-(DPAC) were obtained with scale bars of $40 \mu \mathrm{m}$ and $4 \mu \mathrm{m}$, as shown in fig. S30. Compared to the stretched state, the surfaces in the relaxed state appeared slightly curved without external force exertion. However, both the stretched state and relaxed state showed meaningful changes in microstructure and crystallinity, contributing to the photoluminescence performance of the VIE molecules.

A

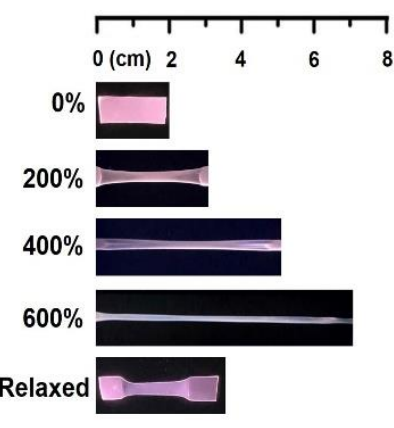

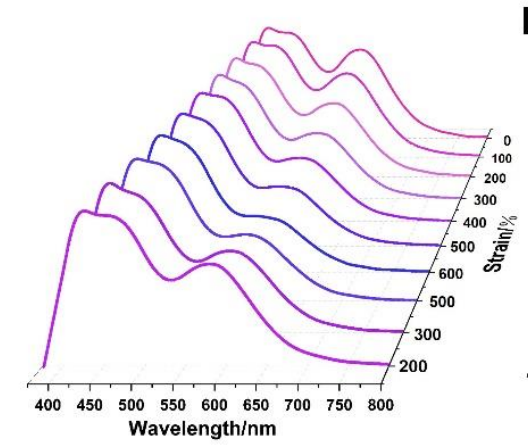

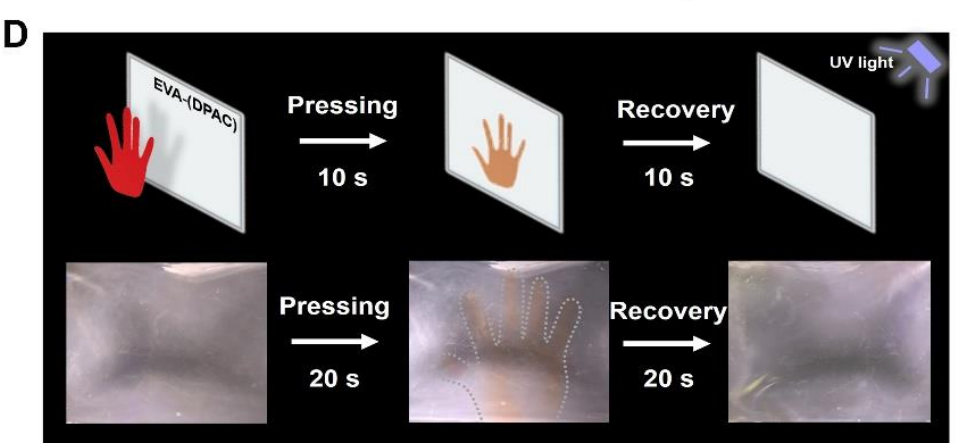

B
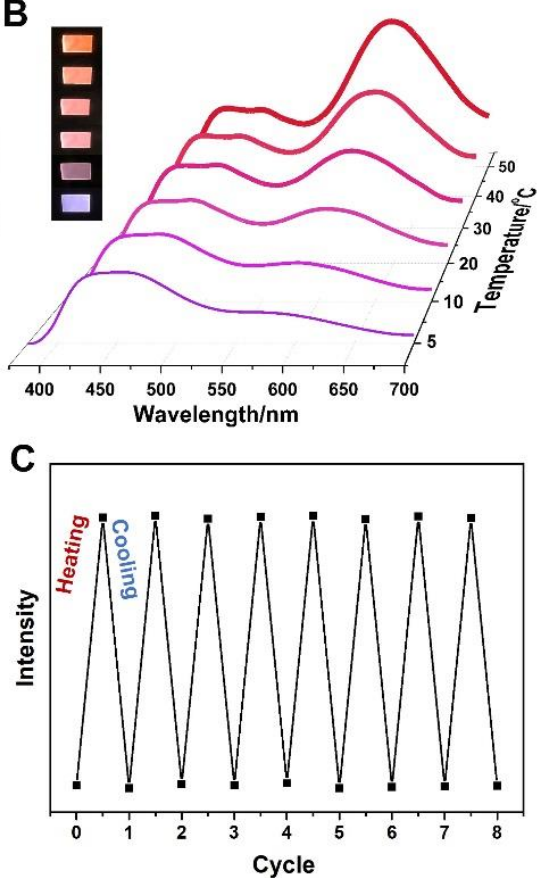


\section{Fig. 5. Stretching properties and temperature response in the doping system of EVA. (A)}

Representative photographs and normalized fluorescence spectra of EVA-(DPAC) in different stretching states and relaxation states $\left(\lambda_{\mathrm{ex}}=365 \mathrm{~nm}\right)$. The line color represents the luminescence color of the related blend films. (B) Normalized fluorescence spectra of EVA-(DPAC) with increasing temperatures from 5 to $50^{\circ} \mathrm{C}\left(\lambda_{\mathrm{ex}}=365 \mathrm{~nm}\right)$. Inset: representative photographs under $365 \mathrm{~nm}$ excitation. (C) Fluorescence emission intensities at $600 \mathrm{~nm}$ upon heating and cooling an EVA-(DPAC) film from 5 to $50^{\circ} \mathrm{C}$ for 8 cycles. (D) Schematic illustration of the temperature response and photographs of the EVA-(DPAC) film at $10^{\circ} \mathrm{C}$, as generated by palm pressing under $365 \mathrm{~nm}$ excitation.

Additionally, the blend films of EVA with VIE molecules also exhibited a precise thermal response with temperature changes from 5 to $50^{\circ} \mathrm{C}$; these films were more sensitive than poly(E-caprolactone). As shown in Fig. 5B and fig. S31, owing to the facilitation of the intramolecular motions of DPAC and DPAC-CN, the normalized fluorescence curves represented multicolor fluorescence from light blue to orange red (pale green to orange red for EVA-(DPAC-CN)). The corresponding CIE coordinate values, as matched with the exact temperatures, are shown in fig. S32 and table S4. The blend films of EVA exhibited rapid nonlinear responses of approximately 125 s upon constant increasing temperature (fig. S33). And the films recovered to their original fluorescence colors faster than poly( $\varepsilon$-caprolactone) from $50^{\circ} \mathrm{C}$ to room temperature, as shown in movie S4. The films exhibited excellent reversibility upon heating and cooling for 8 cycles (Fig. 5C). Furthermore, this significant thermal response in EVA even responded to body temperature exposure by palm pressing. As shown in Fig. 5D, EVA-(DPAC) was processed into a large piece of blend film. The original fluorescence color was recorded at $10^{\circ} \mathrm{C}$ under UV light excitation. However, the fluorescence color of the pressed palm-shaped area turned orange upon $365 \mathrm{~nm}$ irritation. Once the pressing stopped, the fluorescence color recovered quickly without any traces of its previous pressed color. This phenomenon implied that the blend films of EVA with VIE molecules could be utilized as sensitive temperature detectors that can recognize human body temperature.

\section{Conclusion}


In summary, we synthesized two VIE molecules, DPAC and DPAC-CN, with very special vibration-induced emission properties, and these VIE molecules were simply doped in poly( $(\varepsilon-$ caprolactone) and EVA systems to obtain functional optical materials. We systematically explored the photoluminescent properties of the two obtained polymer systems, paving the way for disclosing macrocosmic changes in polymer systems based on the continuous configurations of the VIE molecules. This research has not only reported continuous photoluminescent changes but also established relationships between different forces, temperatures and photoluminescence behaviors. The nature of poly( $\varepsilon$-caprolactone $)$ and the EVA system have been intuitively amplified based on their photoluminescent properties. We believe that the visualization of polymer microcosmos will greatly affect the development of polymer materials. Furthermore, this study has provided an outstanding strategy for elucidating new understandings of polymer systems in the near future.

\section{Supplementary Information:}

Synthetic route and supplementary figures/tables are available in Supplementary Information.

\section{Acknowledgements:}

We gratefully acknowledge the financial support from the National Natural Science Foundation of China (NSFC 21788102, 22020102006, and 21871083), Project supported by Shanghai Municipal Science and Technology Major Project (Grant No. 2018SHZDZX03), Program of Shanghai Academic/Technology Research Leader (20XD1421300), 'Shu Guang' project supported by Shanghai Municipal Education Commission and Shanghai Education Development Foundation (19SG26), the Innovation Program of Shanghai Municipal Education Commission (2017-01-07-00-02-E00010), and the Fundamental Research Funds for the Central Universities.

\section{Competing interests:}

The authors declare no other competing interests. 


\section{References:}

[1] C. Chen, Z. Chi, K. C. Chong, A. S. Batsanov, Z. Yang, Z. Mao, Z. Yang, B. Liu, Carbazole isomers induce ultralong organic phosphorescence. Nat. Mater. 20, 175-180 (2021).

[2] R. Kabe, C. Adachi, Organic long persistent luminescence. Nature 550, 384-387 (2017).

[3] S. Khasminskaya, F. Pyatkov, K. Słowik, S. Ferrari, O. Kahl, V. Kovalyuk, P. Rath, A. Vetter, F. Hennrich, M. M. Kappes, G. Gol'tsman, A. Korneev, C. Rockstuhl, R. Krupke, W. H. Pernice, Fully integrated quantum photonic circuit with an electrically driven light source. Nat. Photon. 10, 727-732 (2016).

[4] H. Uoyama, K. Goushi, K. Shizu, H. Nomura, C. Adachi, Highly efficient organic lightemitting diodes from delayed fluorescence. Nature 492, 234-238 (2012).

[5] F. Zhang, Q. Shen, X. Shi, S. Li, W. Wang, Z. Luo, G. He, P. Zhang, P. Tao, C. Song, W. Zhang, D. Zhang, T. Deng, W. Shang, Infrared detection based on localized modification of morpho butterfly wings. Adv. Mater. 27, 1077-1082 (2015).

[6] C. Liu, Z. Fan, Y. Tan, F. Fan, F. Xu, Tunable structural color patterns based on the visiblelight-responsive dynamic diselenide metathesis. Adv. Mater. 32, 1907569 (2020).

[7] Y. Qi, L. Chu, W. Niu, B. Tang, S. Wu, W. Ma, S. Zhang, New encryption strategy of photonic crystals with bilayer inverse heterostructure guided from transparency response. Adv. Funct. Mater. 29, 1903743 (2019).

[8] H. S. Lee, T. S. Shim, H. Hwang, S. Yang, S. H. Kim, Colloidal photonic crystals toward structural color palettes for security materials. Chem. Mater. 25, 2684 (2013).

[9] Z. Chi, X. Zhang, B. Xu, X. Zhou, C. Ma, Y. Zhang, S. Liu, J. Xu, Recent advances in organic mechanofluorochromic materials. Chem. Soc. Rev., 41, 3878-3896 (2012).

[10]P. Xue, J, Ding, P, Wang, R. Lu, Recent progress in the mechanochromism of phosphorescent organic molecules and metal complexes. J. Mater. Chem. C 4, 6688-6706 (2016).

[11]W. Fan, J. Zeng, Q. Gan, D. Ji, H. Song, W. Liu, L. Shi, L. Wu, Iridescence-controlled and flexibly tunable retroreflective structural color film for smart displays. Sci. Adv. 5, eaaw8755 (2019).

[12] J. Chen, F. Ye, Y. Lin, Z. Chen, S. Liu, J. Yin, Vinyl-functionalized multicolor benzothiadiazoles: Design, synthesis, crystal structures and mechanically-responsive performance. Sci China Chem 62, 440-450 (2019).

[13]M. Li, S. Berritt, C. Wang, X. Yang, Y. Liu, S. Sha, B. Wang, R. Wang, X. Gao, Z. Li, X. Fan, Y. Tao, P. J. Walsh, Sulfenate anions as organocatalysts for benzylic chloromethyl coupling polymerization via $\mathrm{C}=\mathrm{C}$ bond formation. Nat. Commun. 9, 1754 (2018).

[14] D. -H. Lien, S. Z. Uddin, M. Yeh, M. Amani, H. Kim, J. W. Ager III, E. Yablonovitch, A. Javey, Electrical suppression of all nonradiative recombination pathways in monolayer semiconductors. Science 364, 468-471 (2019).

[15]S. Ji, F. Fan, C. Sun, Y. Yu, H. Xu, Visible light-induced plasticity of shape memory polymers. ACS Appl. Mater. Interfaces 9, 33169-33175 (2017).

[16] S. Lu, L. Sui, M. Wu, S. Zhu, X. Yong, B. Yang, Graphitic nitrogen and high-crystalline triggered strong photoluminescence and room-temperature ferromagnetism in carbonized polymer dots. Adv. Sci. 6, 1801192 (2019). 
[17] T. Xiao, W. Zhong, L. Zhou, L. Xu, X. Sun, R. B. Elmes, X. Hu, L. Wang, Artificial lightharvesting systems fabricated by supramolecular host-guest interactions. Chinese. Chem. Lett. 30, 31-36 (2019).

[18]X. Sun, D. Liu, D. Tian, X. Zhang, W. Wu, W. Wan, The introduction of the Barbier reaction into polymer chemistry. Nat. Commun. 8, 1210 (2017).

[19]O. Bolton, K. Lee, H. J. Kim, K. Y. Lin, J. Kim, Activating efficient phosphorescence from purely organic materials by crystal design. Nat. Chem. 3, 205-210 (2011).

[20]C. Shi, Q. Zhang, C. Yu, S. Rao, S. Yang, H. Tian, D. Qu, An ultrastrong and highly stretchable polyurethane elastomer enabled by a zipper-like ring-sliding effect. Adv. Mater. 32, 2000345 (2020).

[21]Q. Zhang, C. Shi, D. Qu, Y. Long, B. L. Feringa, H. Tian, Exploring a naturally tailored small molecule for stretchable, self-healing, and adhesive supramolecular polymers. Sci. $A d v$. 4, eaat8192 (2018).

[22] J. Liu, N. Wang, Y. Yu, Y. Yan, H. Zhang, J. Li, J. Yu, Carbon dots in zeolites: A new class of thermally activated delayed fluorescence materials with ultralong lifetimes. Sci. Adv. 3, e1603171 (2017).

[23] Y. Sagara, S. Yamane, M. Mitani, C. Weder, T. Kato, Mechanoresponsive luminescent molecular assemblies: an emerging class of materials. Adv. Mater. 28, 1073-1095 (2016).

[24] Y. Sagara, M. Karman, E. Verde-Sesto, K. Matsuo, Y. Kim, N. Tamaoki, C. Weder, Rotaxanes as mechanochromic fluorescent force transducers in polymers. J. Am. Chem. Soc. 140, 1584-1587 (2018).

[25]Z. Zhang, Y. Wu, K. Tang, C. Chen, J. Ho, J. Su, H. Tian, P. Chou, Excited-state conformational/electronic responses of saddle-shaped $N, N^{\prime}$-disubstituteddihydrodibenzo[ $[a, c]$ phenazines: wide-tuning emission from red to deep blue and white light combination. J. Am. Chem. Soc. 137, 8509-8520 (2015).

[26] W. Chen, C. Chen, Z. Zhang, Y. Chen, W. Chao, J. Su, H. Tian, P. Chou, Snapshotting the excited-state planarization of chemically locked $N, N^{\prime}$-disubstituted dihydrodibenzo[a,c]phenazines. J. Am. Chem. Soc. 139, 1636-1644 (2017).

[27]H. Wang, Z. Zhang, H. Zhou, T. Wang, J. Su, X. Tong, H. Tian, Cu-catalyzed C-H amination/Ullmann $N$-arylation domino reaction: A straightforward synthesis of 9,14diaryl-9,14-dihydrodibenzo[a,c]phenazine. Chem. Commun. 52, 5459-5462 (2016).

[28]Z. Zhang, C. Chen, Y. Chen, Y. Wei, J. Su, H. Tian, P. Chou, Tuning the conformation and color of conjugated polyheterocyclic skeletons by installing ortho-methyl groups. Angew. Chem., Int. Ed. 57, 9880-9884 (2018).

[29]Z. Zhang, W. Song, J. Su, H. Tian, Vibration-induced emission (VIE) of $N, N^{\prime}-$ disubstituted-dihydribenzo[a,c]phenazines: fundamental understanding and emerging applications. Adv. Funct. Mater. 30, 1902803 (2020).

[30]H. Zhou, J. Mei, Y. Chen, C. Chen, W. Chen, Z. Zhang, J. Su, P. Chou, H. Tian, Phenazinebased ratiometric $\mathrm{Hg}^{2+}$ probes with well-resolved dual emissions: a new sensing mechanism by vibration-induced emission (VIE). Small 12, 6542-6546 (2016).

[31]L. Shi, W. Song, C. Lian, W. Chen, J. Mei, J. Su, H. Liu, H. Tian, Dual-emitting dihydrophenazines for highly sensitive and ratiometric thermometry over a wide temperature range. Adv. Opt. Mater. 6, 1800190 (2018). 
[32]W. Huang, L. Sun, Z. Zheng, J. Su, H. Tian, Colour-tunable fluorescence of single molecules based on the vibration induced emission of phenazine. Chem. Commun. 51, 4462-4464 (2015).

[33]H. Wang, Y. Li, Y. Zhang, J. Mei, J. Su, A new strategy for achieving single-molecular white-light emission: using vibration-induced emission (VIE) plus aggregation-induced emission (AIE) mechanisms as a two-pronged approach. Chem. Commun. 55, 1879-1882 (2019).

[34]J. Wang, X. Yao, Y. Liu, H. Zhou, W. Chen, G. Sun, J. Su, X. Ma, H. Tian, Tunable photoluminescence including white-light emission based on noncovalent interactionlocked $N, N^{\prime}$-disubstituted dihydrodibenzo[a,c]phenazines. Adv. Opt. Mater. 6, 1800074 (2018).

[35] G. Sun, J. Pan, Y. Wu, Y. Liu, W. Chen, Z. Zhang, J. Su, Supramolecular assembly-driven color-tuning and white-light emission based on crown-ether-functionalized dihydrophenazine. ACS Appl. Mater. Interfaces 12, 10875-10882 (2020).

[36] G. Sun, H. Zhou, Y. Liu, Y. Li, Z. Zhang, J. Mei, J. Su, Ratiometric indicator based on vibration-induced emission for in situ and real-time monitoring of gelation processes. ACS Appl. Mater. Interfaces 10, 20205-20212 (2018).

[37]Z. Zhang, G. Sun, W. Chen, J. Su, H. Tian, The endeavor of vibration-induced emission (VIE) for dynamic emissions. Chem. Sci. 11, $7525-7537$ (2020).

[38]Z. Huang, T. Jiang, J. Wang, X. Ma, H. Tian, Real-time visual monitoring of kinetically controlled self-assembly. Angew. Chem., Int. Ed. 60, 2855-2860 (2020).

[39]Z. Jiang, Y. Wang, L. Fu, B. Whiteside, J. Wyborn, K. Norris, Z. Wu, P. Coates, Y. Men, Tensile deformation of oriented poly( $\varepsilon$-caprolactone) and its miscible blends with poly(vinyl methyl ether). Macromolecules 46, 6981-6990 (2013).

[40]T. Defize, J. -M. Thomassin, M. Alexandre, B. Gilbert, R. Riva, C. Jérôme, Comprehensive study of the thermo-reversibility of Diels-Alder based PCL polymer networks. Polymer 84, 234-242 (2016).

[41]A. Zubkiewicz, A. Szymczyk, S. Paszkiewicz, R. Jędrzejewski, E. Piesowicz, J. Siemiński, Ethylene vinyl acetate copolymer/halloysite nanotubes nanocomposites with enhanced mechanical and thermal properties. J Appl. Polym. Sci. 137, 49135 (2020).

[42]C. Hoffendahl, S. Duquesne, G. Fontaine, F. Taschner, M. Mezger, S. Bourbigot, Decomposition mechanism of fire retarded ethylene vinyl acetate elastomer (EVA) containing aluminum trihydroxide and melamine. Polym. Degrad. Stab. 113, 168-179 (2015). 